\title{
Deterministic Concurrent Strategies
}

\author{
Glynn Winskel \\ Computer Laboratory, University of Cambridge, UK
}

\begin{abstract}
Nondeterministic concurrent strategies - those strategies compatible with copy-cat behaving as identity w.r.t. composition - have been characterized as certain maps of event structures. This leads to a bicategory of general concurrent games in which the maps are nondeterministic concurrent strategies. This paper explores the important sub-bicategory of deterministic concurrent strategies. It is shown that deterministic strategies in a game can be identified with certain subgames, with the benefit that the bicategory of deterministic games becomes equivalent to a technically-simpler order-enriched category. Via a characterization, deterministic strategies are shown to coincide with the receptive ingenuous strategies of Melliès and Mimram, providing an external justification for their approach, as yielding the most general deterministic concurrent strategies for which copy-cat behaves as identity. Deterministic strategies determine closure operators, following Abramsky and Melliès. Known subcategories appear as special cases: Berry's order-enriched category of $d I$-domains and stable functions arises as a full subcategory in which the games comprise solely of Player moves; the 'simple games' of Hyland et al., a basis for much of game semantics, form a subcategory in which the games permit no concurrency, Player-Opponent moves alternate and Opponent always moves first. ${ }^{1}$ Finally, winning strategies are considered.
\end{abstract}

\section{Introduction}

This article brings the experience of concurrency (event structures, stable families, their techniques and constructions originally used in the semantics of process languages [2]) to bear on the theory of games. It considers a very general definition of 2-party concurrent games in which Player (more accurately thought of as a team of players) competes against Opponent (a team of opponents) in a potentially highly-distributed fashion, without for instance insisting on the alternation of Player and Opponent moves.

Two-party games and strategies are represented as event structures with polarity, in which polarities distinguish the moves of Player and Opponent-cf. [3]. A total map of event structures with polarity with codomain $A$ can be understood as a pre-strategy in a game $A$ - the map ensures that Player and Opponent respect the constraints of the game. Following Joyal, a pre-strategy from a game $A$ to a game $B$ is understood as a pre-strategy in a composite game got by setting the dual game of $A$, reversing the roles of Player and Opponent, in parallel

\footnotetext{
1 The results on deterministic strategies are reported without proofs in [1].
} 
with $B$. From this general scheme concurrent strategies - pre-strategies for which copy-cat strategies behave as identities w.r.t. composition of pre-strategies - have recently been characterized as those pre-strategies which satisfy the two conditions of receptivity and innocence [1].

The major contribution of this paper is a thorough exploration, with full proofs, of this result's consequences for the case of deterministic strategies of which there is already a significant history within semantics. It gives proofs of necessary and sufficient conditions for copy-cat strategies to be deterministicshows why they are not in general - proves deterministic strategies compose, and that they are necessarily mono as maps into games. The later implies that we can equivalently view deterministic strategies in a game $A$ as certain subfamilies of configurations of $A$. A characterization of precisely which subfamilies arise from deterministic strategies recovers the receptive ingenuous strategies of Melliès and Mimram [4]; their receptive ingenuous strategies are revealed as precisely those deterministic pre-strategies for which copy-cat strategies behave as identities - a satisfying convergence with Melliès' programme of 'asynchronous games' [3].

\section{Event structures and stable families}

An event structure comprises $(E$, Con, $\leq)$, consisting of a set $E$, of events which are partially ordered by $\leq$, the causal dependency relation, and a nonempty consistency relation Con consisting of finite subsets of $E$, which satisfy

$$
\begin{aligned}
& \left\{e^{\prime} \mid e^{\prime} \leq e\right\} \text { is finite for all } e \in E, \\
& \{e\} \in \text { Con for all } e \in E, \\
& Y \subseteq X \in \text { Con } \Longrightarrow Y \in \text { Con, and } \\
& X \in \text { Con } \& e \leq e^{\prime} \in X \Longrightarrow X \cup\{e\} \in \text { Con. }
\end{aligned}
$$

The (finite) configurations, $\mathcal{C}(E)$, of an event structure $E$ consist of those finite subsets $x \subseteq E$ which are

Consistent: $x \in$ Con, and

Down-closed: $\forall e, e^{\prime} . e^{\prime} \leq e \in x \Longrightarrow e^{\prime} \in x$.

Two events which are both consistent and incomparable w.r.t. causal dependency in an event structure are regarded as concurrent. In games the relation of immediate dependency $e \rightarrow e^{\prime}$, meaning $e$ and $e^{\prime}$ are distinct with $e \leq e^{\prime}$ and no event in between, will play a very important role. For $X \subseteq E$ we write $[X]$ for $\left\{e \in E \mid \exists e^{\prime} \in X . e \leq e^{\prime}\right\}$, the down-closure of $X$; note if $X \in$ Con, then $[X] \in$ Con.

Operations such as synchronized parallel composition are awkward to define directly on the simple event structures above. It is useful to broaden event structures to stable families, where operations are often carried out more easily, and then turned into event structures by the operation Pr below.

A stable family comprises $\mathcal{F}$, a nonempty family of finite subsets, called configurations, which satisfy:

Completeness: $\forall Z \subseteq \mathcal{F} . Z \uparrow \Longrightarrow \cup Z \in \mathcal{F}$; 
Coincidence-freeness: For all $x \in \mathcal{F}, e, e^{\prime} \in x$ with $e \neq e^{\prime}$,

$$
\exists y \in \mathcal{F} . y \subseteq x \&\left(e \in y \Longleftrightarrow e^{\prime} \notin y\right) ;
$$

Stability: $\forall x, y \in \mathcal{F} . x \uparrow y \Longrightarrow x \cap y \in \mathcal{F}$.

Above, $Z \uparrow$ means $\exists x \in \mathcal{F} \forall z \in Z$. $z \subseteq x$, and expresses the compatibility of $Z$ in $\mathcal{F}$; we use $x \uparrow y$ for $\{x, y\} \uparrow$. We call elements of $\cup \mathcal{F}$ events of $\mathcal{F}{ }^{2}$

Proposition 1. Let $x$ be a configuration of a stable family $\mathcal{F}$. For $e, e^{\prime} \in x$ define

$$
e^{\prime} \leq_{x} \text { e iff } \forall y \in \mathcal{F} . y \subseteq x \& e \in y \Longrightarrow e^{\prime} \in y .
$$

When $e \in x$ define the prime configuration

$$
[e]_{x}=\bigcap\{y \in \mathcal{F} \mid y \subseteq x \& e \in y\} .
$$

Then $\leq_{x}$ is a partial order and $[e]_{x}$ is a configuration such that

$$
[e]_{x}=\left\{e^{\prime} \in x \mid e^{\prime} \leq_{x} e\right\} .
$$

Moreover the configurations $y \subseteq x$ are exactly the down-closed subsets of $\leq_{x}$.

Proposition 2. Let $\mathcal{F}$ be a stable family. Then, $\operatorname{Pr}(\mathcal{F})=_{\text {def }}(P$, Con, $\leq)$ is an event structure where:

$$
\begin{aligned}
& P=\left\{[e]_{x} \mid e \in x \& x \in \mathcal{F}\right\}, \\
& Z \in \text { Con iff } Z \subseteq P \& \bigcup Z \in \mathcal{F} \text { and }, \\
& p \leq p^{\prime} \text { iff } p, p^{\prime} \in P \& p \subseteq p^{\prime} .
\end{aligned}
$$

A (partial) map of stable families $f: \mathcal{F} \rightarrow \mathcal{G}$ is a partial function $f$ from the events of $\mathcal{F}$ to the events of $\mathcal{G}$ such that for all configurations $x \in \mathcal{F}$,

$$
f x \in \mathcal{G} \&\left(\forall e_{1}, e_{2} \in x . f\left(e_{1}\right)=f\left(e_{2}\right) \Longrightarrow e_{1}=e_{2}\right) .
$$

Maps of event structures are maps of their stable families of configurations. Maps compose as functions. We say a map is total when it is total as a function. Say a total map of event structures is rigid when it preserves causal dependency.

$\operatorname{Pr}$ is the right adjoint of the "inclusion" functor, taking an event structure $E$ to the stable family $\mathcal{C}(E)$. The unit of the adjunction $E \rightarrow \operatorname{Pr}(\mathcal{C}(E))$ takes an event $e$ to the prime configuration $[e]={ }_{\operatorname{def}}\left\{e^{\prime} \in E \mid e^{\prime} \leq e\right\}$. The counit max : $\mathcal{C}(\operatorname{Pr}(\mathcal{F})) \rightarrow \mathcal{F}$ takes prime configuration $[e]_{x}$ to $e$.

Definition 1. Let $\mathcal{F}$ be a stable family. We use $x-c y$ to mean $y$ covers $x$ in $\mathcal{F}$, i.e. $x \subset y$ in $\mathcal{F}$ with nothing in between, and $x \stackrel{e}{e} \subset y$ to mean $x \cup\{e\}=y$ for $x, y \in \mathcal{F}$ and event $e \notin x$. We sometimes use $x \stackrel{e}{-}$, expressing that event $e$ is enabled at configuration $x$, when $x \stackrel{e}{-} c y$ for some $y$. W.r.t. $x \in \mathcal{F}$, write $[e)_{x}=\operatorname{def}\left\{e^{\prime} \in E \mid e^{\prime} \leq_{x} e \& e^{\prime} \neq e\right\}$, so, for example, $[e)_{x} \stackrel{e}{-}[e]_{x}$. The relation of immediate dependence of event structures generalizes: with respect to $x \in \mathcal{F}$, the relation $e \rightarrow_{x} e^{\prime}$ means $e \leq_{x} e^{\prime}$ with $e \neq e^{\prime}$ and no event in between.

${ }^{2}$ We can extend families of finite configurations, $\mathcal{F}$, to families where configurations may be infinite, $\mathcal{F}^{\infty}$, taking $x \in \mathcal{F}^{\infty}$ iff there is a directed subset $S \subseteq \mathcal{F}$ s.t. $x=\bigcup S$. 


\section{Process operations}

\subsection{Products}

Let $\mathcal{A}$ and $\mathcal{B}$ be stable families with events $A$ and $B$, respectively. Their product, the stable family $\mathcal{A} \times \mathcal{B}$, has events comprising pairs in $A \times{ }_{*} B={ }_{\text {def }}\{(a, *) \mid a \in A\} \cup$ $\{(a, b) \mid a \in A \& b \in B\} \cup\{(*, b) \mid b \in B\}$, the product of sets with partial functions, with (partial) projections $\pi_{1}$ and $\pi_{2}$-treating $*$ as 'undefined'- with configurations $x \in \mathcal{A} \times \mathcal{B}$ iff

$$
\begin{aligned}
& x \text { is a finite subset of } A \times_{*} B \text { s.t. } \pi_{1} x \in \mathcal{A} \& \pi_{2} x \in \mathcal{B}, \\
& \forall e, e^{\prime} \in x . \pi_{1}(e)=\pi_{1}\left(e^{\prime}\right) \text { or } \pi_{2}(e)=\pi_{2}\left(e^{\prime}\right) \Rightarrow e=e^{\prime}, \& \\
& \forall e, e^{\prime} \in x . e \neq e^{\prime} \Rightarrow \exists y \subseteq x . \pi_{1} y \in \mathcal{A} \& \pi_{2} y \in \mathcal{B} \&\left(e \in y \Longleftrightarrow e^{\prime} \notin y\right) .
\end{aligned}
$$

Right adjoints preserve products. Consequently we obtain a product of event structures $A$ and $B$ by first regarding them as stable families $\mathcal{C}(A)$ and $\mathcal{C}(B)$, forming their product $\mathcal{C}(A) \times \mathcal{C}(B), \pi_{1}, \pi_{2}$, and then constructing the event structure

$$
A \times B=_{\text {def }} \operatorname{Pr}(\mathcal{C}(A) \times \mathcal{C}(B))
$$

and its projections as $\Pi_{1}=\mathrm{def} \pi_{1} \max$ and $\Pi_{2}=\operatorname{def} \pi_{2} \max$.

\subsection{Restriction}

The restriction of $\mathcal{F}$ to a subset of events $R$ is the stable family $\mathcal{F} \uparrow R=$ def $\{x \in \mathcal{F} \mid x \subseteq R\}$. Defining $E \uparrow R$, the restriction of an event structure $E$ to a subset of events $R$, to have events $E^{\prime}=\{e \in E \mid[e] \subseteq R\}$ with causal dependency and consistency induced by $E$, we obtain $\mathcal{C}(E \uparrow R)=\mathcal{C}(E) \uparrow R$.

Proposition 3. Let $\mathcal{F}$ be a stable family and $R$ a subset of its events. Then, $\operatorname{Pr}(\mathcal{F} \uparrow R)=\operatorname{Pr}(\mathcal{F}) \uparrow \max ^{-1} R$.

\subsection{Synchronized compositions}

Synchronized parallel compositions are obtained as restrictions of products to those events which are allowed to synchronize or occur asynchronously. For example, the synchronized composition of Milner's CCS on stable families $\mathcal{A}$ and $\mathcal{B}$ (with labelled events) is defined as $\mathcal{A} \times \mathcal{B} \uparrow R$ where $R$ comprises events which are pairs $(a, *),(*, b)$ and $(a, b)$, where in the latter case the events $a$ of $\mathcal{A}$ and $b$ of $\mathcal{B}$ carry complementary labels. Similarly, synchronized compositions of event structures $A$ and $B$ are obtained as restrictions $A \times B \uparrow R$. By Proposition 3, we can equivalently form a synchronized composition of event structures by forming the synchronized composition of their stable families of configurations, and then obtaining the resulting event structure - this has the advantage of eliminating superfluous events earlier. 


\subsection{Projection}

Event structures support a simple form of hiding. Let $(E, \leq$, Con $)$ be an event structure. Let $V \subseteq E$ be a subset of 'visible' events. Define the projection of $E$ on $V$, to be $E \downarrow V={ }_{\text {def }}\left(V, \leq_{V}, \operatorname{Con}_{V}\right)$, where $v \leq_{V} v^{\prime}$ iff $v \leq v^{\prime} \& v, v^{\prime} \in V$ and $X \in \operatorname{Con}_{V}$ iff $X \in \operatorname{Con} \& X \subseteq V$.

\section{Event structures with polarities}

We shall represent both a game and a strategy in a game as an event structure with polarity, which comprises $(E, p o l)$ where $E$ is an event structure with a polarity function pol $: E \rightarrow\{+,-\}$ ascribing a polarity + (Player) or - (Opponent) to its events. The events correspond to (occurrences of) moves. Maps of event structures with polarity are maps of event structures which preserve polarity.

\subsection{Operations}

Dual The dual, $E^{\perp}$, of an event structure with polarity $E$ comprises a copy of the event structure $E$ but with a reversal of polarities. It obviously extends to a functor. Write $\bar{e} \in E^{\perp}$ for the event complementary to $e \in E$ and vice versa.

Simple parallel composition This operation simply juxtaposes two event structures with polarity. Let $\left(A, \leq_{A}, \operatorname{Con}_{A}, \operatorname{pol}_{A}\right)$ and $\left(B, \leq_{B}, \operatorname{Con}_{B}, \operatorname{pol}_{B}\right)$ be event structures with polarity. The events of $A \| B$ are $(\{1\} \times A) \cup(\{2\} \times B)$, their polarities unchanged, with: the only relations of causal dependency given by $(1, a) \leq\left(1, a^{\prime}\right)$ iff $a \leq_{A} a^{\prime}$ and $(2, b) \leq\left(2, b^{\prime}\right)$ iff $b \leq_{B} b^{\prime}$; a subset of events $C$ is consistent in $A \| B$ iff $\{a \mid(1, a) \in C\} \in \operatorname{Con}_{A}$ and $\{b \mid(2, b) \in C\} \in \operatorname{Con}_{B}$. The operation extends to a functor - put the two maps in parallel. The empty event structure with polarity $\varnothing$ is the unit w.r.t. $\|$.

\section{Pre-strategies}

Let $A$ be an event structure with polarity, thought of as a game; its events stand for the possible occurrences of moves of Player and Opponent and its causal dependency and consistency relations the constraints imposed by the game. A pre-strategy in $A$ is a total map $\sigma: S \rightarrow A$ from an event structure with polarity $S$. A pre-strategy represents a nondeterministic play of the game - all its moves are moves allowed by the game and obey the constraints of the game; the concept will later be refined to that of strategy (and winning strategy in Section 8.1).

Let $A$ and $B$ be event structures with polarity. Following Joyal [5], a prestrategy from $A$ to $B$ is a pre-strategy in $A^{\perp} \| B$, so a total map $\sigma: S \rightarrow A^{\perp} \| B$. It thus determines a span

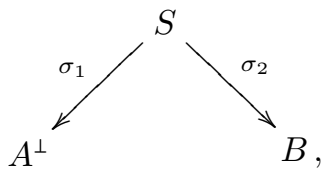


of event structures with polarity where $\sigma_{1}, \sigma_{2}$ are partial maps. In fact, a prestrategy from $A$ to $B$ corresponds to such spans where for all $s \in S$ either, but not both, $\sigma_{1}(s)$ or $\sigma_{2}(s)$ is defined. Two pre-strategies will be essentially the same when they are isomorphic as spans. We write $\sigma \cong \tau$, for pre-strategies $\sigma$ and $\tau$ from $A$ to $B$ when their spans are isomorphic. We write $\sigma: A \nrightarrow B$ to express that $\sigma$ is a pre-strategy from $A$ to $B$. Note a pre-strategy in a game $A$ coincides with a pre-strategy from the empty game $\sigma: \varnothing \rightarrow A$.

\subsection{Composing pre-strategies}

Consider two pre-strategies $\sigma: A \nrightarrow B$ and $\tau: B \nrightarrow C$ as spans:
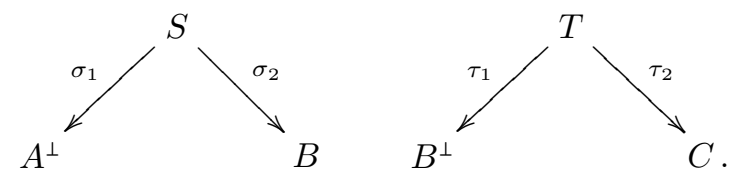

We show how to define their composition $\tau \odot \sigma: A \rightarrow C$ as the result of a synchronized composition, followed by projection to hide internal synchronization events. We first form the synchronized composition of $S$ and $T$ by restricting the product $S \times T$, with projections $\Pi_{1}: S \times T \rightarrow S$ and $\Pi_{2}: S \times T \rightarrow T$, to allow only those synchronizations associated with complementary events, of different polarities, in $B$ and $B^{\perp}$. Specifically, the synchronized composition is $S \times T \uparrow R_{0}$ where

$$
\begin{aligned}
R_{0}= & \left\{p \in S \times T \mid \sigma_{1} \Pi_{1}(p) \text { is defined } \& \Pi_{2}(p) \text { is undefined }\right\} \cup \\
& \left\{p \in S \times T \mid \tau_{2} \Pi_{2}(p) \text { is defined } \& \Pi_{1}(p) \text { is undefined }\right\} \cup \\
& \left\{p \in S \times T \mid \sigma_{2} \Pi_{1}(p)=\overline{\tau_{1} \Pi_{2}(p)} \text { with both defined }\right\} .
\end{aligned}
$$

We define $T \odot S=$ def $\left(S \times T \uparrow R_{0}\right) \downarrow V$ where

$V=\left\{p \in S \times T \uparrow R_{0} \mid \sigma_{1} \Pi_{1}(p)\right.$ is defined $\} \cup\left\{p \in S \times T \uparrow R_{0} \mid \tau_{2} \Pi_{2}(p)\right.$ is defined $\}$.

Finally, the composition $\tau \odot \sigma$ is defined to be the span

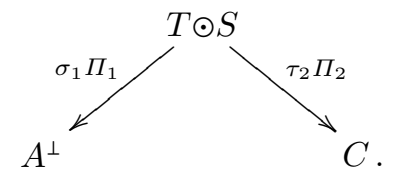

As remarked in Section 3.3, the same construction is achieved by first forming the synchronized composition of the stable families $\mathcal{C}(S)$ and $\mathcal{C}(T)$ (it is this description we shall use in proofs):

Proposition 4. The composition $T \odot S=\operatorname{Pr}(\mathcal{C}(S) \times \mathcal{C}(T) \uparrow R) \downarrow V$, where

$$
\begin{aligned}
R= & \left\{(s, *) \mid s \in S \& \sigma_{1}(s) \text { is defined }\right\} \cup\left\{(*, t) \mid t \in T \& \tau_{2}(t) \text { is defined }\right\} \cup \\
& \left\{(s, t) \mid s \in S \& t \in T \& \sigma_{2}(s)=\overline{\tau_{1}(t)} \text { with both defined }\right\} .
\end{aligned}
$$


The span $\tau \odot \sigma$ comprises maps $v_{1}: T \odot S \rightarrow A^{\perp}$ and $v_{2}: T \odot S \rightarrow C$, which on events $p$ of $T \odot S$ act so $v_{1}(p)=\sigma_{1}(s)$ when $\max (p)=(s, *)$ and $v_{2}(p)=\tau_{2}(t)$ when $\max (p)=(*, t)$, and are undefined elsewhere.

The natural isomorphism $S \times(T \times U) \cong(S \times T) \times U$, associated with the product of event structures $S, T, U$, restricts to the required isomorphism of spans as the synchronizations involved in successive compositions are disjoint:

Proposition 5. Let $\sigma: A \rightarrow B, \tau: B \rightarrow C$ and $v: C \rightarrow D$ be pre-strategies. The two compositions $v \odot(\tau \odot \sigma)$ and $(v \odot \tau) \odot \sigma$ are isomorphic.

\subsection{Concurrent copy-cat}

Identities w.r.t. composition are given by copy-cat strategies. Let $A$ be an event structure with polarity. The copy-cat strategy from $A$ to $A$ is an instance of a pre-strategy, so a total map $\gamma_{A}: \mathrm{CC}_{A} \rightarrow A^{\perp} \| A$. It describes a concurrent, or distributed, strategy based on the idea that Player moves, of +ve polarity, always copy previous corresponding moves of Opponent, of -ve polarity.

For $c \in A^{\perp} \| A$ we use $\bar{c}$ to mean the corresponding copy of $c$, of opposite polarity, in the alternative component, i.e. $\overline{(1, a)}=(2, \bar{a})$ and $\overline{(2, a)}=(1, \bar{a})$. Define $\mathbb{C C}_{A}$ to comprise the event structure with polarity $A^{\perp} \| A$ together with extra causal dependencies $\bar{c} \leq_{\mathrm{CC}_{A}} c$ for all events $c$ with $\operatorname{pol}_{A^{\perp} \| A}(c)=+$.

Proposition 6. Let $A$ be an event structure with polarity. Then event structure with polarity $\mathrm{CC}_{A}$ is an event structure. Moreover, $x \in \mathcal{C}\left(\mathrm{CC}_{A}\right)$ iff $x \in \mathcal{C}\left(A^{\perp} \| A\right) \& \forall c \in x . p o l_{A^{\perp} \| A}(c)=+\Longrightarrow \bar{c} \in x$.

The copy-cat pre-strategy $\gamma_{A}: A \rightarrow A$ is defined to be the map $\gamma_{A}: \mathbb{C}_{A} \rightarrow$ $A^{\perp} \| A$ where $\gamma_{A}$ is the identity on the common set of events.

\section{Strategies}

The main result of [1], presented summarily, is that two conditions on prestrategies, receptivity and innocence, are necessary and sufficient for copy-cat to behave as identity w.r.t. the composition of pre-strategies. Receptivity ensures an openness to all possible moves of Opponent. Innocence restricts the behaviour of Player; Player may only introduce new relations of immediate causality of the form $\ominus \rightarrow \oplus$ beyond those imposed by the game.

Receptivity. A pre-strategy $\sigma$ is receptive iff $\sigma x \stackrel{a}{\complement} \subset \operatorname{pol}_{A}(a)=-\Rightarrow \exists ! s \in$ S. $x \stackrel{s}{\subset} \& \&(s)=a$.

Innocence. A pre-strategy $\sigma$ is innocent when it is both + -innocent: if $s \rightarrow s^{\prime} \& \operatorname{pol}(s)=+$ then $\sigma(s) \rightarrow \sigma\left(s^{\prime}\right)$, and --innocent: if $s \rightarrow s^{\prime} \& \operatorname{pol}\left(s^{\prime}\right)=-$ then $\sigma(s) \rightarrow \sigma\left(s^{\prime}\right)$.

Theorem 1. Let $\sigma: A \rightarrow B$ be pre-strategy. Copy-cat behaves as identity w.r.t. composition, i.e. $\sigma \circ \gamma_{A} \cong \sigma$ and $\gamma_{B} \circ \sigma \cong \sigma$, iff $\sigma$ is receptive and innocent. 


\subsection{The bicategory of concurrent games and strategies}

Theorem 1 motivates the definition of a strategy as a pre-strategy which is receptive and innocent. In fact, we obtain a bicategory, Games, in which the objects are event structures with polarity - the games, the arrows from $A$ to $B$ are strategies $\sigma: A \nrightarrow B$ and the 2-cells are maps of spans. The vertical composition of 2 -cells is the usual composition of maps of spans. Horizontal composition is given by the composition of strategies $\odot$ (which extends to a functor on 2-cells via the functoriality of synchronized composition).

\section{Deterministic strategies}

\subsection{Definition}

We say an event structure with polarity $S$ is deterministic iff

$$
\forall X \subseteq_{\text {fin }} S . N e g[X] \in \operatorname{Con}_{S} \Longrightarrow X \in \operatorname{Con}_{S},
$$

where $N e g[X]==_{\operatorname{def}}\left\{s^{\prime} \in S \mid \operatorname{pol}\left(s^{\prime}\right)=-\& \exists s \in X . s^{\prime} \leq s\right\}$. In other words, $S$ is deterministic iff any finite set of moves is consistent when it causally depends only on a consistent set of opponent moves. Say a strategy $\sigma: S \rightarrow A$ is deterministic if $S$ is deterministic.

Lemma 1. An event structure with polarity $S$ is deterministic iff

$$
\forall s, s^{\prime} \in S, x \in \mathcal{C}(S) . \quad x \stackrel{s}{\subset} \& x \stackrel{s^{\prime}}{\complement} \& \operatorname{pol}(s)=+\Longrightarrow x \cup\left\{s, s^{\prime}\right\} \in \mathcal{C}(S) .
$$

Proof. "Only if": Assume $S$ is deterministic, $x \stackrel{s}{\subset}, x \stackrel{s^{\prime}}{\subset}$ and $\operatorname{pol}(s)=+$. Take $X={ }_{\text {def }} x \cup\left\{s, s^{\prime}\right\}$. Then $N e g[X] \subseteq x \cup\{s\}$ so $N e g[X] \in \operatorname{Con}_{S}$. As $S$ is deterministic, $X \in \mathrm{Con}_{S}$ and being down-closed $X=x \cup\left\{s, s^{\prime}\right\} \in \mathcal{C}(S)$.

"If": Assume $S$ satisfies the property stated above in the proposition. Let $X \subseteq_{\text {fin }}$ $S$ with $N e g[X] \in \operatorname{Con}_{S}$. Then the down-closure $[N e g[X]] \in \mathcal{C}(S)$. Clearly $[N e g[X]] \subseteq$ $[X]$ where all events in $[X] \backslash[N e g[X]]$ are necessarily +ve. Suppose, to obtain a contradiction, that $X \notin \operatorname{Con}_{S}$. Then there is a maximal $z \in \mathcal{C}(S)$ such that

$$
[N e g[X]] \subseteq z \subseteq[X]
$$

and some $e \in[X] \backslash z$, necessarily $+\mathrm{ve}$, for which $[e) \subseteq z$. Take a covering chain

$$
[e) \stackrel{s_{1}}{\longrightarrow} z_{1} \stackrel{s_{2}}{\subset} \cdots \stackrel{s_{k}}{\complement} z_{k}=z .
$$

As $[e) \stackrel{e}{-} c[e]$ with $e+$ ve, by repeated use of the property of the lemmaillustrated below-we obtain $z \stackrel{e}{\complement} \subset z^{\prime}$ in $\mathcal{C}(S)$ with $[N e g[X]] \subseteq z^{\prime} \subseteq[X]$, which contradicts the maximality of $z$.

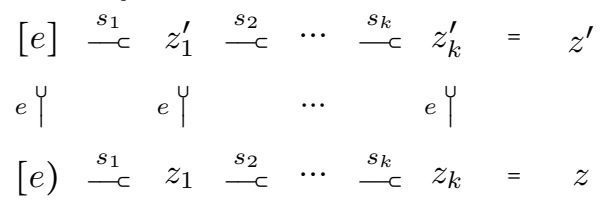


So, above, an event structure with polarity can fail to be deterministic in two ways, either with $\operatorname{pol}(s)=\operatorname{pol}\left(s^{\prime}\right)=+$ or with $\operatorname{pol}(s)=+\& \operatorname{pol}\left(s^{\prime}\right)=-$. In general for an event structure with polarity $A$ the copy-cat strategy can fail to be deterministic in either way, illustrated in the examples below.

Example 1. (i) Take $A$ to consist of two +ve events and one -ve event, with any two but not all three events consistent. The construction of $\mathrm{CC}_{A}$ is pictured:

$$
\begin{aligned}
\ominus & \rightarrow \oplus \\
A^{\perp} \ominus & \rightarrow \oplus A \\
\oplus & \leftarrow \ominus
\end{aligned}
$$

Here $\gamma_{A}$ is not deterministic: take $x$ to be the set of all three -ve events in $\mathbb{C C}_{A}$ and $s, s^{\prime}$ to be the two +ve events in the $A$ component.

(ii) Take $A$ to consist of two events, one +ve and one -ve event, inconsistent with each other. The construction $\mathrm{CC}_{A}$ :

$$
\begin{aligned}
A^{\perp} & \ominus \rightarrow \oplus A \\
& \oplus \leftarrow \ominus
\end{aligned}
$$

To see $\mathbb{C C}_{A}$ is not deterministic, take $x$ to be the singleton set consisting e.g. of the -ve event on the left and $s, s^{\prime}$ to be the +ve and -ve events on the right.

\subsection{The bicategory of deterministic strategies}

We first characterize those games for which copy-cat is deterministic; they only allow immediate conflict between events of the same polarity.

Lemma 2. Let $A$ be an event structure with polarity. The copy-cat strategy $\gamma_{A}$ is deterministic iff A satisfies

$$
\forall x \in \mathcal{C}(A) . x \stackrel{a}{ } \subset \& x \stackrel{a^{\prime}}{\subset} \subset \operatorname{pol}(a)=+\& \operatorname{pol}\left(a^{\prime}\right)=-\Longrightarrow x \cup\left\{a, a^{\prime}\right\} \in \mathcal{C}(A) .
$$

Proof. "Only if": Suppose $x \in \mathcal{C}(A)$ with $x \stackrel{a}{\complement} \subset$ and $x \stackrel{a^{\prime}}{\complement}$ where $\operatorname{pol}(a)=+$ and $\operatorname{pol}\left(a^{\prime}\right)=-$. Construct $y={ }_{\operatorname{def}}\{(1, \bar{b}) \mid b \in x\} \cup\{(1, \bar{a})\} \cup\{(2, b) \mid b \in x\}$. Then $y \in \mathcal{C}\left(\mathrm{CC}_{A}\right)$ with $y \stackrel{(2, a)}{\complement}$ and $y \stackrel{\left(2, a^{\prime}\right)}{\complement}$, by Proposition 6(ii). Assuming $\mathbb{C C}_{A}$ is deterministic, we obtain $y \cup\left\{(2, a),\left(2, a^{\prime}\right)\right\} \in \mathcal{C}\left(\mathbb{C C}_{A}\right)$, so $y \cup\left\{(2, a),\left(2, a^{\prime}\right)\right\} \in \mathcal{C}\left(A^{\perp} \| A\right)$. This entails $x \cup\left\{a, a^{\prime}\right\} \in \mathcal{C}(A)$, as required to show $(\ddagger)$.

"If": Assume $A$ satisfies ( $\ddagger)$. It suffices to show for $X \subseteq_{\text {fin }} C_{A}$, with $X$ downclosed, that $N e g[X] \in \operatorname{Con}_{\mathrm{CC}_{A}}$ implies $X \in \mathrm{Con}_{\mathrm{CC}_{A}}$. Recall $Z \in \operatorname{Con}_{\mathrm{CC}_{A}}$ iff $Z \in$ $\operatorname{Con}_{A^{\perp} \| A}$.

Let $X \subseteq_{\text {fin }} \mathbb{C C}_{A}$ with $X$ down-closed. Assume $N e g[X] \in \operatorname{Con}_{\mathrm{CC}_{A}}$. Observe

(i) $\{c \mid c \in X \& \operatorname{pol}(c)=-\} \subseteq N e g[X]$ and

(ii) $\{\bar{c} \mid c \in X \& \operatorname{pol}(c)=+\} \subseteq N e g[X]$ as by Proposition $6, X$ being down-closed must contain $\bar{c}$ if it contains $c$ with $\operatorname{pol}(c)=+$. 
Consider $X_{2}=\operatorname{def}\{a \mid(2, a) \in X\}$. Then $X_{2}$ is a finite down-closed subset of $A$. From (i),

$$
X_{2}^{-}=_{\text {def }}\left\{a \in X_{2} \mid \operatorname{pol}(a)=-\right\} \in \operatorname{Con}_{A} .
$$

From (ii),

$$
X_{2}^{+}={ }_{\text {def }}\left\{a \in X_{2} \mid \operatorname{pol}(a)=+\right\} \in \operatorname{Con}_{A} .
$$

We show ( $\ddagger$ ) implies $X_{2} \in \operatorname{Con}_{A}$.

Define $z^{-}=_{\operatorname{def}}\left[X_{2}^{-}\right]$and $z^{+}={ }_{\operatorname{def}}\left[X_{2}^{+}\right]$. Being down-closures of consistent sets, $z^{-}, z^{+} \in \mathcal{C}(A)$. We show $z^{-} \uparrow z^{+}$in $\mathcal{C}(A)$. First note $z^{-} \cap z^{+} \in \mathcal{C}(A)$. If $a \in z^{-} \backslash z^{-} \cap z^{+}$ then $\operatorname{pol}(a)=-$; otherwise, if $\operatorname{pol}(a)=+$ then $a \in z^{+}$a well as $a \in z^{-}$making $a \in z^{-} \cap z^{+}$, a contradiction. Similarly, if $a \in z^{+} \backslash z^{-} \cap z^{+}$then $\operatorname{pol}(a)=+$. We can form covering chains

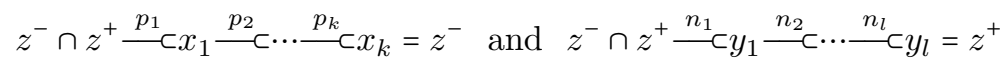

where each $p_{i}$ is + ve and each $n_{j}$ is - ve.

Consequently, by repeated use of $(\ddagger)$, we obtain $x_{k} \cup y_{l} \in \mathcal{C}(A)$, i.e. $z^{+} \cup z^{-} \epsilon$ $\mathcal{C}(A)$, as is illustrated below. But $X_{2} \subseteq z^{+} \cup z^{-}$, so $X_{2} \in \operatorname{Con}_{A}$. A similar argument shows $X_{1}={ }_{\text {def }}\left\{a \in A^{\perp} \mid(1, a) \in X\right\} \in \operatorname{Con}_{A^{\perp}}$. It follows that $X \in \operatorname{Con}_{A^{\perp} \| A}$, so $X \in \mathrm{Con}_{\mathrm{CC}_{A}}$ as required.

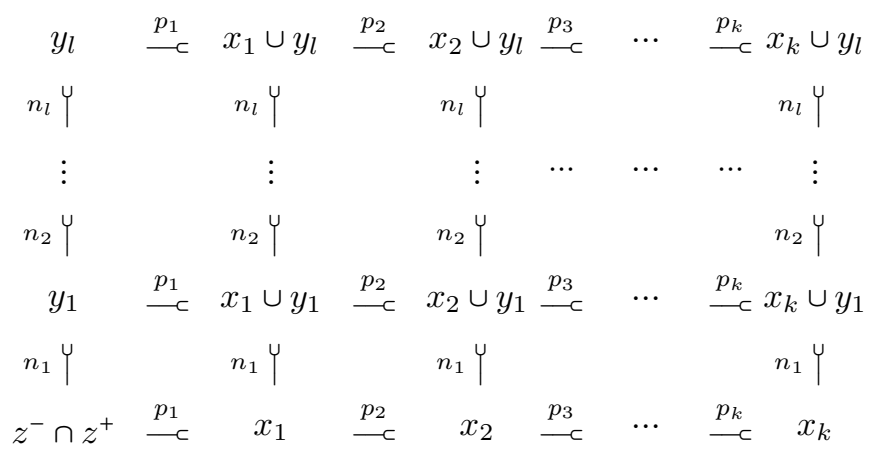

Via the next lemma, when games satisfy $(\ddagger)$ we can simplify the condition for a strategy to be deterministic.

Lemma 3. Let $\sigma: S \rightarrow A$ be a strategy. Suppose $x \stackrel{s}{\longrightarrow} \subset y \& x \stackrel{s^{\prime}}{\complement} y^{\prime} \& \operatorname{pol}_{S}(s)=-$. Then, $\sigma y \uparrow \sigma y^{\prime}$ in $\mathcal{C}(A) \Longrightarrow y \uparrow y^{\prime}$ in $\mathcal{C}(S)$.

Proof. Assume $\sigma y \uparrow \sigma y^{\prime}$ in $\mathcal{C}(A)$, so $\sigma y^{\prime} \stackrel{\sigma(s)}{\complement} \sigma y \cup \sigma y^{\prime}$ in $\mathcal{C}(A)$. As $\sigma(s)$ is -ve, by receptivity, there is a unique $s^{\prime \prime} \in S$, necessarily -ve, such that $\sigma\left(s^{\prime \prime}\right)=\sigma(s)$ and $y^{\prime} \stackrel{s^{\prime \prime}}{\subset} \subset \cup\left\{s^{\prime}, s^{\prime \prime}\right\}$ in $\mathcal{C}(S)$. In particular, $x \cup\left\{s^{\prime}, s^{\prime \prime}\right\} \in \mathcal{C}(S)$. By --innocence, we cannot have $s^{\prime} \rightarrow s^{\prime \prime}$, so $x \cup\left\{s^{\prime \prime}\right\} \in \mathcal{C}(S)$. But now $x \stackrel{s}{\sim}$ and $x \stackrel{s^{\prime \prime}}{ } \subset$ with $\sigma(s)=\sigma\left(s^{\prime \prime}\right)$ and both $s, s^{\prime \prime}$-ve and hence $s^{\prime \prime}=s$ by the uniqueness part of receptivity. We conclude that $x \cup\left\{s^{\prime}, s\right\} \in \mathcal{C}(S)$ so $y \uparrow y^{\prime}$. 
Corollary 1. Assume A satisfies ( $f$ ) of Lemma 2. A strategy $\sigma: S \rightarrow A$ is deterministic iff for all + ve events $s, s^{\prime} \in S$ and configurations $x \in \mathcal{C}(S)$,

$$
x \stackrel{s}{\subset} \subset \& \stackrel{s^{\prime}}{\subset} \Longrightarrow x \cup\left\{s, s^{\prime}\right\} \in \mathcal{C}(S) .
$$

Proof. "Only if": clear. "If": Let $x \stackrel{s}{\complement}$ and $x \stackrel{s^{\prime}}{\subset}$ where $\operatorname{pol}_{S}(s)=+$. For $S$ to be deterministic we require $x \cup\left\{s, s^{\prime}\right\} \in \mathcal{C}(S)$. The above assumption ensures this when $\operatorname{pol}_{S}\left(s^{\prime}\right)=+$. Otherwise $\operatorname{pol}_{S}\left(s^{\prime}\right)=-$ with $\sigma x \stackrel{\sigma(s)}{\subset}$ and $\sigma x \stackrel{\sigma\left(s^{\prime}\right)}{\subset}$. As $A$ satisfies $(\ddagger), \sigma x \cup \sigma(s), \sigma\left(s^{\prime}\right) \in \mathcal{C}(A)$. Now by Lemma $3, x \cup\left\{s, s^{\prime}\right\} \in \mathcal{C}(S)$.

Lemma 4. The composition $\tau \odot \sigma$ of deterministic strategies $\sigma$ and $\tau$ is deterministic.

Proof. Let $\sigma: S \rightarrow A^{\perp} \| B$ and $\tau: T \rightarrow B^{\perp} \| C$ be deterministic strategies. The composition $T \odot S$ is constructed as $\operatorname{Pr}(\mathcal{C}(T) \odot \mathcal{C}(S)) \downarrow V$, a synchronized composition of event structures $S$ and $T$ projected to visible events $e \in V$ where $\max (e)$ has the form $(s, *)$ or $(*, t)$.

We first note a fact about the effect of internal, or "invisible," events not in $V$ on configurations of $\mathcal{C}(T) \odot \mathcal{C}(S)$. If

$$
z \stackrel{(s, t)}{\complement} w \& z \stackrel{\left(s^{\prime}, t^{\prime}\right)}{\complement} w^{\prime} \& w \uparrow w^{\prime}
$$

within $\mathcal{C}(T) \odot \mathcal{C}(S)$, then either

$$
\pi_{1} z \stackrel{s}{\subset} \subset \pi_{1} w \& \pi_{1} z \stackrel{s^{\prime}}{\llcorner} \subset \pi_{1} w^{\prime} \& \pi_{1} w \uparrow \pi_{1} w^{\prime},
$$

within $\mathcal{C}(S)$, or

$$
\pi_{2} z \stackrel{t}{\complement} \subset \pi_{2} w \& \pi_{2} z \stackrel{t^{\prime}}{\complement} \subset \pi_{2} w^{\prime} \& \pi_{2} w \uparrow \pi_{2} w^{\prime},
$$

within $\mathcal{C}(T)$. Assume (1). If $t=t^{\prime}$ then $\sigma(s)=\overline{\tau(t)}=\overline{\tau\left(t^{\prime}\right)}=\sigma\left(s^{\prime}\right)$ and we obtain (2) as $\sigma$ is a map of event structures. Similarly if $s=s^{\prime}$ then (3). Supposing $s \neq s^{\prime}$ and $t \neq t^{\prime}$ then if both (2) and (3) failed we could construct a configuration $z^{\prime}={ }_{\text {def }} z \cup\left\{(s, t),\left(s^{\prime}, t\right)\right\}$ of $\mathcal{C}(T) \odot \mathcal{C}(S)$, contradicting (1); it is easy to check that $z^{\prime}$ is a configuration of the product $\mathcal{C}(S) \times \mathcal{C}(T)$ and its events are clearly within the restriction used in defining the synchronized composition.

We now show the impossibility of (2) and (3), and so (1). Assume (2) (case (3) is similar). One of $s$ or $s^{\prime}$ being +ve would contradict $S$ being deterministic. Suppose otherwise, that both $s$ and $s^{\prime}$ are -ve. Then, because $\sigma$ is a strategy, by Lemma 3, we have

$$
\sigma_{2} \pi_{1} w \uparrow \sigma_{2} \pi_{1} w^{\prime}
$$

in $\mathcal{C}(B)$. Also, then both $t$ and $t^{\prime}$ are + ve ensuring $\pi_{2} w \uparrow \pi_{2} w^{\prime}$ in $\mathcal{C}(T)$, as $T$ is deterministic. This entails

$$
\tau_{1} \pi_{2} w \uparrow \tau_{1} \pi_{2} w^{\prime}
$$


in $\mathcal{C}\left(B^{\perp}\right)$. But $\sigma_{2} \pi_{1} w$ and $\tau_{1} \pi_{2} w$, respectively $\sigma_{2} \pi_{1} w^{\prime}$ and $\tau_{1} \pi_{2} w^{\prime}$, are the same configurations on the common event structure underlying $B$ and $B^{\perp}$, of which we have obtained contradictory statements of compatibility.

As (1) is impossible, it follows that

$$
z \stackrel{(s, t)}{\subset} w \& z \stackrel{\left(s^{\prime}, t^{\prime}\right)}{\complement} w^{\prime} \Longrightarrow w \uparrow w^{\prime}
$$

within $\mathcal{C}(T) \odot \mathcal{C}(S)$.

Finally, we can show that $\tau \odot \sigma$ is deterministic. Suppose $x \stackrel{p}{-} \subset y$ and $x \stackrel{p^{\prime}}{\longrightarrow} \subset y^{\prime}$ in $\mathcal{C}(T \odot S)$ with $\operatorname{pol}(p)=+$. Then,

$$
\bigcup x \stackrel{e_{1}}{\complement} \subset z_{1} \stackrel{e_{2}}{\subset} \subset \stackrel{e_{k}}{\longrightarrow} \subset z_{k}=\bigcup y \text { and } \bigcup x \stackrel{e_{1}^{\prime}}{\subset} \subset z_{1}^{\prime} \stackrel{e_{2}^{\prime}}{\complement} \subset \stackrel{e_{l}^{\prime}}{\longrightarrow} \subset z_{l}^{\prime}=\bigcup y^{\prime}
$$

in $\mathcal{C}(T) \odot \mathcal{C}(S)$, where $e_{k}=\max (p)$ and $e_{l}^{\prime}=\max \left(p^{\prime}\right)$, and the events $e_{i}$ and $e_{j}^{\prime}$ otherwise have the form $e_{i}=\left(s_{i}, t_{i}\right)$, when $1 \leq i<k$, and $e_{j}^{\prime}=\left(s_{j}^{\prime}, t_{j}^{\prime}\right)$, when $1 \leq j<l$. By repeated use of (4) we obtain $z_{k-1} \uparrow z_{l-1}^{\prime}$. (The argument is like that ending the proof of Lemma 2, though with the minor difference that now we may have $e_{i}=e_{j}^{\prime}$.) We obtain $w==_{\text {def }} z_{k-1} \cup z_{l-1}^{\prime} \in \mathcal{C}(T) \odot \mathcal{C}(S)$ with $w \stackrel{e_{k}}{\subset}$ and $w \stackrel{e_{l}^{\prime}}{\subset}$ and $\operatorname{pol}\left(e_{k}\right)=+$.

Now, $w \cup\left\{e_{k}, e_{l}^{\prime}\right\} \in \mathcal{C}(T) \odot \mathcal{C}(S)$ provided $w \cup\left\{e_{k}, e_{l}^{\prime}\right\} \in \mathcal{C}(S) \times \mathcal{C}(T)$. Inspect the definition of configurations of the product of stable families in Section 3.1. If $e_{k}$ and $e_{l}^{\prime}$ have the form $(s, *)$ and $\left(s^{\prime}, *\right)$ respectively, then determinacy of $S$ ensures that the projection $\pi_{1} w \cup\left\{s, s^{\prime}\right\} \in \mathcal{C}(S)$ whence $w \cup\left\{e_{k}, e_{l}^{\prime}\right\}$ meets the conditions needed to be in $\mathcal{C}(S) \times \mathcal{C}(T)$. Similarly, $w \cup\left\{e_{k}, e_{l}^{\prime}\right\} \in \mathcal{C}(S) \times \mathcal{C}(T)$ if $e_{k}$ and $e_{l}^{\prime}$ have the form $(*, t)$ and $\left(*, t^{\prime}\right)$. Otherwise one of $e_{k}$ and $e_{l}^{\prime}$ has the form $(s, *)$ and the other $(*, t)$. In this case again an inspection of the definition of configurations of the product yields $w \cup\left\{e_{k}, e_{l}^{\prime}\right\} \in \mathcal{C}(S) \times \mathcal{C}(T)$. Forming the set of primes of $w \cup\left\{e_{k}, e_{l}^{\prime}\right\}$ in $V$ we obtain $x \cup\left\{p, p^{\prime}\right\} \in \mathcal{C}(T \odot S)$.

This establishes that $T \odot S$ is deterministic.

We thus obtain a sub-bicategory DGames of Games; its objects satisfy ( $\ddagger)$ of Lemma 2 and its maps are deterministic strategies.

\subsection{A category of deterministic strategies}

In fact, DGames is equivalent to an order-enriched category via the following lemma. It says deterministic strategies in a game $A$ are essentially certain subfamilies of configurations $\mathcal{C}(A)$, for which we give a characterization.

Lemma 5. A deterministic strategy is injective on configurations (i.e., is mono as a map of event structures).

Proof. Let $\sigma: S \rightarrow A$ be a deterministic strategy. We show

$$
x \supseteq z-c y \& \sigma y \subseteq \sigma x \Longrightarrow y \subseteq x,
$$

for $x, y, z \in \mathcal{C}(S)$, by induction on $|x \backslash z|$. 
Suppose $x \supseteq z \stackrel{e}{\complement} c y$ and $\sigma y \subseteq \sigma x$. There are $x_{1}$ and event $e_{1} \in S$ such that $z \stackrel{e_{1}}{\neg} x_{1} \subseteq x$. If $\sigma\left(e_{1}\right)=\sigma(e)$ then $e_{1}, e$ have the same polarity; if - ve $e_{1}=e$, by receptivity; if + ve $e_{1}=e$, by determinacy with the local injectivity of $\sigma$. Either way $y \subseteq x$. Suppose $\sigma\left(e_{1}\right) \neq \sigma(e)$.

Case $\operatorname{pol}\left(e_{1}\right)=\operatorname{pol}(e)=+$ : As $\sigma$ is deterministic, $e_{1}$ and $e$ are concurrent giving $x_{1} \stackrel{e}{\subset} y \cup\left\{e_{1}\right\}$. By induction we obtain $y \cup\left\{e_{1}\right\} \subseteq x$.

Case $\operatorname{pol}(e)=-:$ As both $\sigma z \stackrel{\sigma\left(e_{1}\right)}{\complement} \sigma x$ and $\sigma z \stackrel{\sigma(e)}{\complement} \sigma y$ and $\sigma y \subseteq \sigma x$, we have $\sigma\left(e_{1}\right)$ and $\sigma(e)$ concurrent in $A$. In particular, $\sigma x_{1} \stackrel{\sigma(e)}{\subset}$ where $\operatorname{pol}(\sigma(e))=-$. By receptivity, there is $e^{\prime}$ such that $x_{1} \stackrel{e^{\prime}}{\subset}$ with $\sigma\left(e^{\prime}\right)=\sigma(e)$. Because of innocence, we cannot have $e_{1} \rightarrow e^{\prime}$ in $S$, so $e_{1}$ and $e^{\prime}$ are concurrent. Consequently $z \stackrel{e^{\prime}}{ }$. But then $e^{\prime}=e$ by the uniqueness part of receptivity. We deduce that $e_{1}$ and $e$ are concurrent yielding $x_{1} \stackrel{e}{\subset} \subset y \cup\left\{e_{1}\right\}$, and by induction $y \cup\left\{e_{1}\right\} \subseteq x$. The case where $\operatorname{pol}\left(e_{1}\right)=-$ is very similar.

Another, simpler induction on $|y \backslash z|$ now yields

$$
x \supseteq z \subseteq y \& \sigma y \subseteq \sigma x \Longrightarrow y \subseteq x,
$$

for $x, y, z \in \mathcal{C}(S)$, from which the result follows.

A deterministic strategy $\sigma: S \rightarrow A$ determines, as the image of the configurations $\mathcal{C}(S)$, a subfamily $F=$ def $\sigma \mathcal{C}(S)$ of configurations of $\mathcal{C}(A)$, satisfying:

reachability: $\varnothing \in F$ and if $x \in F$ there is a covering chain $\varnothing \stackrel{a_{1}}{\subset} \subset x_{1} \stackrel{a_{2}}{\subset} \cdots \stackrel{a_{k}}{\subset} \subset x_{k}=x$ within $F$;

determinacy: If $x \stackrel{a}{\subset}$ and $x \stackrel{a^{\prime}}{\complement}$ in $F$ with $\operatorname{pol}_{A}(a)=+$, then $x \cup\left\{a, a^{\prime}\right\} \in F$;

receptivity: If $x \in F$ and $x \stackrel{a}{\subset} \subset$ in $\mathcal{C}(A)$ and $\operatorname{pol}_{A}(a)=-$, then $x \cup\{a\} \in F$;

+-innocence: If $x \stackrel{a}{\longrightarrow} \subset x_{1} \stackrel{a^{\prime}}{\longrightarrow} \subset$ \& $\operatorname{pol}_{A}(a)=+$ in $F$ and $x \stackrel{a^{\prime}}{ } \subset$ in $\mathcal{C}(A)$, then $x \stackrel{a^{\prime}}{ } \subset$ in $F$ (here receptivity implies --innocence);

cube: In $F$,

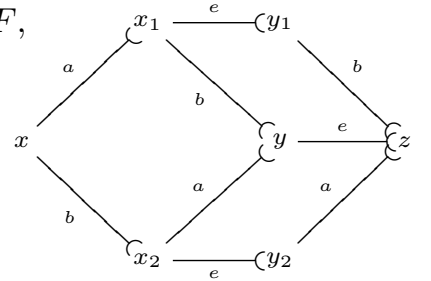

implies

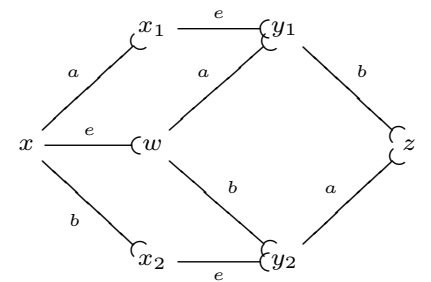

Theorem 2. A subfamily $F \subseteq \mathcal{C}(A)$ satisfies the axioms above iff there is a deterministic strategy $\sigma: S \rightarrow A$ s.t. $F=\sigma \mathcal{C}(S)$, the image of $\mathcal{C}(S)$ under $\sigma$.

Proof. (Sketch) It is routine to check that $F$, the image $\sigma \mathcal{C}(S)$ of a deterministic strategy, satisfies the axioms. Conversely, suppose a subfamily $F \subseteq \mathcal{C}(A)$ satisfies the axioms. We show $F$ is a stable family. First note that from the axioms of determinacy and receptivity we can deduce:

$$
\text { if } x \stackrel{a}{\subset} \text { and } x \stackrel{a^{\prime}}{ } \subset \text { in } F \text { with } x \cup\left\{a, a^{\prime}\right\} \in \mathcal{C}(A) \text {, then } x \cup\left\{a, a^{\prime}\right\} \in F \text {. }
$$


By repeated use of this property, using their reachability, if $x, y \in F$ and $x \uparrow y$ in $\mathcal{C}(A)$ then $x \cup y \in F$; the proof also yields a covering chain from $x$ to $x \cup y$ and from $y$ to $x \cup y$. (In particular, if $x \subseteq y$ in $F$, then there is a covering chain from $x$ to $y$-a fact we shall use shortly.) Thus, if $x \uparrow y$ in $F$ then $x \cup y \in F$. As also $\varnothing \in F$, we obtain Completeness, required of a stable family. Coincidence-freeness is a direct consequence of reachability. Repeated use of the cube axiom yields

Cube: In $F$,

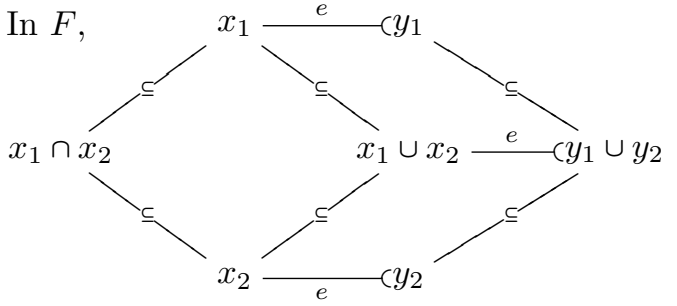

implies

We use Cube to show stability. Assume $v \uparrow w$ in $F$. Let $z \in F$ be maximal s.t. $z \subseteq v, w$. We show $z=v \cap w$. Suppose not. Then, forming covering chains in $F$,

$$
z \stackrel{c_{1}}{\longrightarrow} \subset v_{1} \stackrel{c_{2}}{\complement} \subset \stackrel{c_{k}}{\complement} \subset v_{k}=v \text { and } z \stackrel{d_{1}}{\longleftarrow} \subset w_{1} \stackrel{d_{2}}{\complement} \subset \stackrel{d_{l}}{\longrightarrow} \subset w_{l}=w,
$$

there are $c_{i}$ and $d_{j}$ s.t. $c_{i}=d_{j}$, where we may assume $c_{i}$ is the earliest event to be repeated as some $d_{j}$. Write $e==_{\text {def }} c_{i}=d_{j}$. Now, $v_{i-1} \cap w_{j-1}=z$. Also, being bounded above $v_{i-1} \cup w_{j-1} \in F$ and $v_{i} \cup w_{j} \in F$. We have an instance of Cube: take $x_{1}=v_{i-1}, x_{2}=w_{j-1}, y_{1}=v_{i}$ and $y_{2}=w_{j}$. Hence $z \stackrel{e}{\complement}$ and $z \cup\{e\} \subseteq x, y-$ contradicting the maximality of $z$. Therefore $z=v \cap w$, as required for stability.

Now we can form an event structure $S==_{\text {def }} \operatorname{Pr}(F)$. The inclusion $F \subseteq \mathcal{C}(A)$ induces a total map $\sigma: S \rightarrow A$ for which $F=\sigma \mathcal{C}(S)$. Note that--innocence (viz. if $x \stackrel{a}{ } \subset x_{1} \stackrel{a^{\prime}}{\complement} \subset \& p o l_{A}\left(a^{\prime}\right)=-$ in $F$ and $x \stackrel{a^{\prime}}{ } \subset$ in $\mathcal{C}(A)$, then $x \stackrel{a^{\prime}}{ } \subset$ in $\left.F\right)$ is a direct consequence of receptivity. That $S$ is deterministic follows from determinacy, that $\sigma$ is a strategy from the axioms of receptivity and +-innocence.

We can thus identify deterministic strategies from $A$ to $B$ with subfamilies of $\mathcal{C}\left(A^{\perp} \| B\right)$ satisfying the axioms above. Through this identification we obtain an order-enriched category of deterministic strategies (presented as subfamilies) equivalent to DGames.

\section{Related work}

Ingenuous strategies [4] Via Theorem 2, deterministic concurrent strategies coincide with the receptive ingenuous strategies of Melliès and Mimram.

Stable spans and stable functions [6,7] The sub-bicategory of Games where the events of games are purely +ve is equivalent to the bicategory of stable spans. In this case, strategies correspond to stable spans:

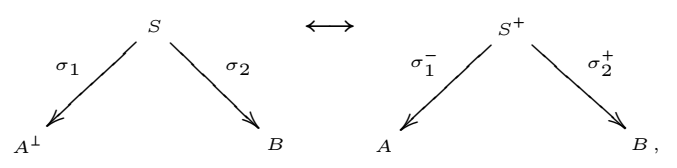


where $S^{+}$is the projection of $S$ to its +ve events; $\sigma_{2}^{+}$is the restriction of $\sigma_{2}$ to $S^{+}$, necessarily a rigid map by innocence; $\sigma_{2}^{-}$is a demand map taking $x \in \mathcal{C}\left(S^{+}\right)$ to $\sigma_{1}^{-}(x)=\sigma_{1}[x]$; here $[x]$ is the down-closure of $x$ in $S$. If we further restrict strategies to be deterministic (and, strictly, event structures to be countable) we obtain a bicategory equivalent to Berry's $d I$-domains and stable functions.

Closure operators $[\mathbf{8}, 4]$ A deterministic strategy $\sigma: S \rightarrow A$ determines a closure operator $\varphi$ on possibly infinite configurations $\mathcal{C}(S)^{\infty}$ : for $x \in \mathcal{C}(S)^{\infty}$,

$$
\varphi(x)=x \cup\{s \in S \mid \operatorname{pol}(s)=+\& \operatorname{Neg}[\{s\}] \subseteq x\} .
$$

Clearly $\varphi$ preserves intersections of configurations and is continuous. The closure operator $\varphi$ on $\mathcal{C}(S)^{\infty}$ induces a partial closure operator $\varphi_{p}$ on $\mathcal{C}(A)^{\infty}$. This in turn determines a closure operator $\varphi_{p}^{\top}$ on $\mathcal{C}(A)^{\infty \top}$, where configurations are extended with a top T, $c f$. [8]: take $y \in \mathcal{C}(A)^{\infty \top}$ to the least, fixed point of $\varphi_{p}$ above $y$, if such exists, and T otherwise.

Simple games $[\mathbf{9}, \mathbf{1 0}]$ The subcategory of "simple games" arises when we restrict DGames to objects and deterministic strategies whose configurations take the form of a tree and polarity alternates on the events of branches which always begin with Opponent.

Extensions Games, such as those of [11,12], allowing copying are being systematized through the use of monads and comonads [10], work now feasible on event structures with symmetry [7].

\subsection{Winning strategies}

A game with losing configurations comprises $G=(A, L)$ where $A$ is an event structure with polarity and $L \subseteq \mathcal{C}(A)^{\infty}$ consists of the losing configurations for Player. A strategy in $G$ is a strategy in $A$. It is regarded as winning if it always prescribes Player moves to avoid ending up in a losing configuration, no matter what the activity or inactivity of Opponent. Formally, a strategy $\sigma: S \rightarrow A$ in $G$ is a winning (for Player) if $\sigma x \notin L$ for all +-maximal configurations $x \in \mathcal{C}(S)^{\infty}$-a configuration $x$ is +-maximal if whenever $x \stackrel{s}{\subset} \subset$ then the event $s$ has -ve polarity. In the special case of a deterministic strategy $\sigma$ in $G$ it is winning iff $\sigma \varphi(x) \notin L$ for all $x \in \mathcal{C}(S)^{\infty}$, where $\varphi$ is the closure operator $\varphi: \mathcal{C}(S)^{\infty} \rightarrow \mathcal{C}(S)^{\infty}$ determined by $\sigma$ or, equivalently, the images under $\sigma$ of fixed points of $\varphi$ lie outside $L$ - see earlier in this section for the definition of the closure operator $\varphi$.

Not all games with losing configurations have winning strategies. Consider the game $A$ consisting of one player move $\oplus$ and one opponent move $\ominus$ inconsistent with each other, with losing configurations $L=\{\varnothing,\{\ominus\}\}$. This game has no winning strategy; any strategy $\sigma: S \rightarrow A$, being receptive, will have an event $s \in S$ with $\sigma(s)=\ominus$, and so the losing $\{s\}$ as a + -maximal configuration.

There is an obvious dual of a game with losing configurations: $(A, L)^{\perp}$ is essentially $\left(A^{\perp}, L^{c}\right)$ where $L^{c}=\mathcal{C}(A)^{\infty} \backslash L$ - strictly, we should take the copy of $L^{c}$ in $\mathcal{C}\left(A^{\perp}\right)^{\infty}$. With some experimentation, one reasonable way to form the parallel composition of two games with losing configurations is to take

$$
\left(A, L_{A}\right) \|\left(B, L_{B}\right)=\left(A\left\|B, L_{A}\right\| L_{B}\right)
$$


where $X \| Y=\{\{1\} \times x \cup\{2\} \times y \mid x \in X \& y \in Y\}$ when $X$ and $Y$ are subsets of configurations; in $G \| H$ to lose is to lose in both games $G$ and $H .{ }^{3}$ We can again follow Joyal and define strategies between games now with losing configurations: a (winning) strategy from $G$ to $H$ is a (winning) strategy in $G^{\perp} \| H$. We compose strategies as before. Copy-cat strategies are winning. The composition of winning strategies is winning. (The proof relies on the observation that a configuration $x$ in $(\mathcal{C}(S) \times \mathcal{C}(T) \uparrow R)^{\infty}$, used in Proposition 4 to obtain the composition of strategies $T$ and $S$, is +-maximal iff its projections $\pi_{1} x$ and $\pi_{2} x$ are +-maximal configurations of $S$ and $T$ respectively.) Defining $G \otimes H=_{\text {def }}\left(G^{\perp} \| H^{\perp}\right)^{\perp}$ we obtain a game where to lose is to lose in either game $G$ or $H$.

\section{Acknowledgment}

Thanks to Nathan Bowler, Pierre Clairambault, Pierre-Louis Curien, Marcelo Fiore, Julian Gutierrez, Jonathan Hayman, Martin Hyland, Paul-André Melliès, Samuel Mimram, Gordon Plotkin, Silvain Rideau and Sam Staton for helpful remarks. The support of a Royal Society Leverhulme Trust Senior Fellowship and Advanced Grant ECSYM of the ERC are acknowledged with gratitude.

\section{References}

1. Rideau, S., Winskel, G.: Concurrent strategies. In: LICS 2011, IEEE Computer Society (2011)

2. Winskel, G.: Event structure semantics for CCS and related languages. In: ICALP'82. Volume 140 of LNCS., Springer (1982)

3. Melliès, P.A.: Asynchronous games 2: The true concurrency of innocence. Theor. Comput. Sci. 358(2-3): 200-228 (2006)

4. Melliès, P.A., Mimram, S.: Asynchronous games : innocence without alternation. In: CONCUR '07. Volume 4703 of LNCS., Springer (2007)

5. Joyal, A.: Remarques sur la théorie des jeux à deux personnes. Gazette des sciences mathématiques du Québec, 1(4) (1997)

6. Saunders-Evans, L., Winskel, G.: Event structure spans for nondeterministic dataflow. Electr. Notes Theor. Comput. Sci. 175(3): 109-129 (2007)

7. Winskel, G.: Event structures with symmetry. Electr. Notes Theor. Comput. Sci. 172: 611-652 (2007)

8. Abramsky, S., Melliès, P.A.: Concurrent games and full completeness. In: LICS '99, IEEE Computer Society (1999)

9. Hyland, M.: Game semantics. In Pitts, A., Dybjer, P., eds.: Semantics and Logics of Computation. Publications of the Newton Institute (1997)

10. Harmer, R., Hyland, M., Melliès, P.A.: Categorical combinatorics for innocent strategies. In: LICS '07, IEEE Computer Society (2007)

11. Hyland, J.M.E., Ong, C.H.L.: On full abstraction for PCF: I, II, and III. Inf. Comput. 163(2): 285-408 (2000)

12. Abramsky, S., Jagadeesan, R., Malacaria, P.: Full abstraction for PCF. Inf. Comput. 163(2): 409-470 (2000)

\footnotetext{
${ }^{3}$ I'm grateful to Nathan Bowler, Pierre Clairambault and Julian Gutierrez for guidance in the definition of parallel composition of games with losing configurations.
} 